\title{
MÉTODO PARA DETERMINAÇÃO DE VALORES NA AVALIAÇÃO IMOBILIÁRIA: COMPARAÇÃO ENTRE O MODELO DE REGRESSÃO LINEAR E LÓGICA FUZZY
}

\author{
Method For Determining Values In Real Estate Appraisal: comparing between \\ Linear Regression Model and Fuzzy Logic
}

\author{
Carolina Scherrer Malaman ${ }^{1}$ \\ Amilton Amorim ${ }^{1}$ \\ 1 Programa de Pós-Graduação em Ciências Cartográficas. Universidade Estadual Paulista - Presidente \\ Prudente - SP. Brasil. cmalaman@gmail.com; amorim@fct.unesp.br
}

\section{Resumo:}

A lógica fuzzy é uma ferramenta apropriada para lidar com variáveis incertas e pode contribuir consideravelmente com as técnicas utilizadas atualmente nas avaliações imobiliárias, já que devido a alguns fatores, como mudanças de compradores e vendedores de um imóvel para outro, as variáveis que definem o valor são difíceis de serem modeladas. Neste contexto, o objetivo deste trabalho foi testar a utilização da lógica fuzzy como uma alternativa para a avaliação de imóveis, propondo um novo método para modelar o mercado imobiliário. Por se tratar de uma técnica de abordagem supervisionada foi necessário um conhecimento aprofundado da área de interesse, visando a escolha das variáveis e a exploração dos dados. Foi utilizada uma amostra de dados reais do município de Álvares Machado-SP, possibilitando a comparação dos resultados obtidos utilizando lógica fuzzy, com os resultados apresentados pelo método tradicional que, na maioria dos casos, é feito por regressão linear. A comparação entre os métodos mostrou melhores resultados para o modelo de regressão. No entanto, o modelo fuzzy mostrou ter um bom potencial para a avaliação de imóveis.

Palavras-chave: Avaliação imobiliária, Lógica fuzzy, Planta de Valores Genéricos.

\begin{abstract}
:
Fuzzy logic is a suitable tool for dealing with uncertain variables and it can contribute considerably with the techniques currently used in real state properties valuation, due to the fact that some variables, which define the value of a property, are difficult to be modeled, for instance the changes of buyers and sellers from a property to another one. Because it is a supervised approach technique was necessary in-depth knowledge of the area of interest, targeting the choice of variables and exploitation of data. In this context, the aim of this study was testing the use of fuzzy logic as an alternative for the assessment of real estate values, proposing a new method to model the real estate market. A sample of real data from the town Álvares Machado-SP was used in the experiments. The results obtained with fuzzy logic were compared with those obtained from a traditional method, which is usually performed using linear regression. The comparison between the methods showed that the best results were obtained with the regression model. However, the fuzzy logic model showed to have good potential for the real estate values assessment.
\end{abstract}

Keywords: Real estate appraisal, Fuzzy Logic, Average Real Estate Price Map. 


\section{Introdução}

No Brasil, com a criação da Lei de Responsabilidade Fiscal-LRF (Brasil, 2000), que disciplina atos administrativos, e o Estatuto da Cidade (Brasil, 2001), que regulamenta instrumentos de desenvolvimento urbano, os municípios passaram a se preocupar mais com o seu sistema cadastral. Assim, tem se tornado cada vez mais necessário aos órgãos administrativos das cidades fazer um mapeamento de toda sua área urbana, o que envolve o levantamento de dados descritivos e geográficos e uma avaliação detalhada dos imóveis, com a finalidade de estabelecer uma base para a cobrança justa de impostos. Na tributação imobiliária urbana essa base é, normalmente, uma Planta de Valores Genéricos - PVG.

A Planta de Valores Genéricos consiste em um documento cartográfico e descritivo que representa a distribuição espacial dos valores dos imóveis em cada região da cidade. Geralmente, os valores são apresentados por face de quadra. Esse documento tem por finalidade não só servir como base de dados para a tributação, mas também para todo o processo de planejamento urbano, como conhecimento da riqueza da cidade e dos bairros, para definições das diretrizes de desenvolvimento previstas no Plano Diretor Municipal e de prioridades de investimentos (Averbeck, 2003).

A Associação Brasileira de Normas Técnicas (ABNT) estabelece, pela NBR 14653 (ABNT, 2011a), as regras específicas para a avaliação de imóveis urbanos, que é uma das etapas para a elaboração da PVG, informando as diretrizes básicas para a realização desse tipo de trabalho. A NBR 14653-1 (ABNT, 2011a) trata dos procedimentos gerais para a avaliação de bens e indica alguns métodos que devem ser utilizados para tal finalidade. A escolha do método mais adequado depende, basicamente, da natureza do bem a ser avaliado, da finalidade da avaliação e da disponibilidade, qualidade e quantidade de dados obtidos no mercado.

Dos métodos propostos pela ABNT, o mais utilizado é o Método Comparativo Direto de Dados do Mercado, no qual o valor do imóvel é obtido comparando-se as características do bem em avaliação com as de outros imóveis semelhantes que foram negociados no mercado. Tendo em vista a dificuldade de se encontrar estes elementos semelhantes, são utilizados métodos que levem à representação do comportamento do mercado imobiliário.

A NBR 14653-2 (ABNT, 2011b) sugere duas metodologias básicas para o tratamento dos dados: o tratamento por fatores de homogeneização e a metodologia científica. A metodologia científica com o uso da regressão linear é adotada pela maioria dos avaliadores e bastante desenvolvida no Brasil, porém outras ferramentas podem ser aplicadas, desde que devidamente justificadas do ponto de vista teórico e prático. Segundo González (2002), a regressão linear apresenta alguns inconvenientes que resultam na diminuição da precisão das estimativas, indicando a necessidade de aperfeiçoamento.

A busca por métodos alternativos justifica-se pela importância econômica e social do mercado imobiliário, e pelas possíveis consequências da imprecisão nas mensurações realizadas nesse campo, ou seja, os prejuízos econômicos e sociais decorrentes dos erros cometidos nas estimativas do valor de mercado dos imóveis. Os modelos de avaliação imobiliária podem ser empregados na definição de planos diretores, em estudos de viabilidade econômica de novas construções, nas estimativas para obtenção de financiamentos, nas desapropriações e na tributação imobiliária, sendo que as avaliações incorretas podem causar erros no planejamento urbano ou na avaliação da viabilidade econômica. 
Diversos trabalhos vêm sendo publicados, propondo alternativas para melhorar as técnicas de avaliação de imóveis, nas diversas áreas relacionadas: Mello e Santello (2012) apresentam um método de apoio à decisão para a avaliação de imóveis utilizando lógica fuzzy, na forma de árvore de decisão; Moraes (2008) utiliza ferramentas de lógica fuzzy para avaliar a qualidade ambiental em áreas urbanas recuperadas e Cay e Iscan (2011) apresentam um método de redistribuição de terra utilizando lógica fuzzy.

Diferentemente da lógica booleana, na lógica fuzzy não existe somente respostas extremas, ela permite atribuir um grau de aproximação da solução, buscando não somente a resposta exata, mas o quanto se aproxima ou não dessa resposta. A lógica fuzzy surgiu há mais de 40 anos visando resolver problemas de controle e automação, porém, devido à adaptabilidade e proximidade com problemas do mundo real, sua utilização foi crescendo com o passar dos anos e se expandindo para diversas aplicações como Geoprocessamento, Medicina, Análise de Riscos, Avaliações, Controle de Qualidade, entre outras.

As estratégias de controle fuzzy imitam um comportamento baseado em regras vindas da experiência do especialista em vez de um controle restrito a modelos matemáticos. Portanto, o modelo fuzzy comporta-se conforme o raciocínio que o especialista utiliza para inferir as regras, baseadas nas informações que eles já conhecem. Assim, os modelos fuzzy podem variar não só de acordo com os fatores que os definem, mas também de acordo com o especialista.

Na avaliação imobiliária, as variáveis (proximidade ao centro, característica da região onde está localizado, padrão do imóvel etc.) que definem o valor, são difíceis de serem estimadas e podem variar de avaliador para avaliador ou de comprador para vendedor, tornando-se variáveis incertas. A lógica fuzzy é uma ferramenta apropriada para lidar com incertezas dessa natureza e pode contribuir com as técnicas atualmente utilizadas no tratamento estatístico para a atribuição dos valores utilizados nas avaliações imobiliárias.

Por se tratar de um método de abordagem supervisionada, a lógica fuzzy obriga um conhecimento aprofundado sobre as informações que se deseja modelar, por exemplo as variáveis que definem o valor, e consequentemente pode trazer uma melhor compreensão quanto a exploração dos dados.

Diante do exposto, a proposta deste trabalho foi testar a utilização da lógica fuzzy como uma alternativa para a avaliação de imóveis, propondo um método para modelar o mercado imobiliário, diferente dos métodos tradicionais, e possibilitando a comparação entre os resultados obtidos com a lógica fuzzy e os resultados obtidos por meio de modelos de regressão para o mesmo conjunto de dados.

\section{Fundamentação Teórica}

\subsection{Avaliação Imobiliária}

A avaliação de imóveis utiliza métodos que visam determinar o valor de mercado de um bem (imóvel). A aplicação do melhor método para se chegar ao valor do imóvel depende das condições que o mercado imobiliário oferece ao avaliador. $\mathrm{O}$ fato que deve ser observado, neste momento, são as informações que se têm disponíveis e a finalidade das avaliações. Assim, podese dizer que a escolha do método para obter o valor de um imóvel é dependente das informações disponíveis e do nível de rigor que se deseja ter (ABNT, 2011b). 
No Brasil, a primeira norma brasileira para avaliação de imóveis urbanos, NB 502, foi publicada em 1977 pela ABNT. Em 1980, esta norma sofreu uma revisão, foi registrada no INMETRO (Instituto Nacional de Metrologia, Normalização e Qualidade Industrial) com nomeação NBR 5676, e tinha como novidade o estabelecimento de níveis de precisão na avaliação. Atualmente, a avaliação de imóveis urbanos no Brasil é normatizada pela NBR 14653-2 (ABNT, 2011b), publicada em maio de 2004 e atualizada em 2011.

A NBR 14653-2 (ABNT, 2011b) tem o objetivo de fixar as diretrizes para a avaliação de imóveis urbanos, quanto à classificação de sua natureza, descrição das atividades básicas, do uso das metodologias, das especificações da avaliação, e suporte para laudos e pareceres técnicos de avaliação. Além disso, a norma apresenta ainda, em seu texto, a descrição de vários métodos possíveis que podem ser aplicados nos processos de avaliação.

\subsection{Lógica fuzzy}

A dificuldade, ou até mesmo a impossibilidade, de se obter todas as informações e de equacionar a realidade imprecisa do mundo real, levou alguns cientistas a propor lógicas alternativas que seriam propícias à representação daquelas situações particulares em que existe a incerteza. Uma dessas proposições é a lógica fuzzy (Azevedo et al., 2000).

O termo fuzzy e a formalização dos conjuntos fuzzy foram introduzidos por Lotfi A. Zadeh, em 1965, quando ele trabalhava com problemas de classificações de conjuntos que não possuíam fronteiras bem definidas, ou seja, a transição entre os conjuntos deveria ser suave e não abrupta. Em muitos problemas de Física e Matemática, não se têm dificuldades em classificar elementos como pertencentes ou não a um determinado conjunto clássico. Dessa forma, dado um conjunto A e um elemento $\mathrm{x}$ do conjunto universo $\mathrm{U}$, consegue-se muitas vezes dizer se $\mathrm{x} \in \mathrm{A}$ ou se $\mathrm{x} \notin$ A.

Afirma-se sem receio, por exemplo, que o número 5 pertence ao conjunto dos números naturais e que o número -5 não pertence a este mesmo conjunto. Este é o caso sobre o qual não se tem dúvida, sendo a lógica booleana devidamente aplicada. No entanto, pode-se discordar quanto ao número 4,5 pertencer ou não ao conjunto dos números "aproximadamente iguais a 5". Neste caso, a resposta não é única e objetiva, pertencer ou não ao conjunto poderá depender do tipo de problema que se está analisando (Ortega, 2001).

Um conjunto fuzzy é caracterizado por uma função de pertinência que mapeia os elementos de um espaço $X$ para um número real no intervalo [0;1], ou formalmente, $\mathrm{A}: \mathrm{X} \rightarrow[0 ; 1]$.

As classes de funções de pertinência mais utilizadas são: linear por partes (triangular e trapezoidal), gaussiana e sigmoidal.

A escolha do formato da função de pertinência mais adequada nem sempre é óbvia, podendo inclusive não estar ao alcance do conhecimento para uma determinada aplicação. No entanto, existem sistemas fuzzy cujos parâmetros das funções de pertinências podem ser bem definidos por especialistas. A escolha de funções triangulares e trapezoidais é a mais comum, pois a ideia de se definir regiões de pertinências total, média e nula é mais intuitiva do que a especificação do valor médio e de dispersão, conceitos esses ligados às funções gaussianas (Benini e Meneguette Junior, 2009).

Os conjuntos fuzzy podem ser associados a variáveis linguísticas que possibilitam descrever as informações de maneira qualitativa, ou seja, seus valores estão relacionados às palavras ou 
frases, não aos números (Benini e Meneguette Junior, 2009). A variável sempre está ligada a um conjunto que determina quais valores ela pode assumir. Por exemplo, para a variável temperatura da água, seus valores podem ser "fria", "morna" e "quente".

A inferência fuzzy é fundamentada sobre a implicação e a composição de regras que, por meio de proposições condicionais do tipo "se" e "então" e com base em variáveis linguísticas, chega-se a um resultado que permite tomar decisão.

A capacidade de descrever ou classificar detalhes de forma gradual, permitindo uma aproximação muito maior da realidade, faz com que a lógica fuzzy possua as mais diversas aplicações que vão desde soluções em desenvolvimento industrial, ciências ambientais até na área de negócios e finanças.

$\mathrm{Na}$ avaliação de imóveis, as características descritivas de um determinado imóvel servem para estimar seu valor final, através de proposições lógicas ou regras linguísticas. Um exemplo dessas regras pode ser: se o imóvel é grande e de luxo, então seu preço é alto. Os termos, grande, luxo e alto são valores possíveis para as variáveis linguísticas, tamanho, padrão e preço, por exemplo, que são atributos qualificativos dos imóveis (Murgel Filho, 2005).

Existe uma relação entre o número de conjuntos que as variáveis de entrada formam e a quantidade de regras necessárias para o melhor entendimento da realidade modelada pelo sistema fuzzy. Quanto maior o número de variáveis ou o número de conjuntos em que são divididas, maior o número de regras que devem ser estabelecidas. Por exemplo, se a variável tamanho for dividida em três conjuntos (grande, médio e pequeno) e a variável padrão em quatro conjuntos (luxo, alto, médio e baixo) o número de regras ideal será $3 * 4=12$.

Obtido um conjunto fuzzy por intermédio do processo de inferência, realiza-se a defuzzificação, ou seja, o estado da variável de saída é transformado para um valor numérico. Sendo assim, a defuzzificação determina os resultados esperados e, portanto, sua adequação ao modelo representado deve ser testada criteriosamente (Runkler, 1997).

Existem vários métodos de defuzzificação, sendo os mais comuns: centroide, centro dos máximos e média dos máximos (Barros; Brassanezi, 2006)

- Centroide: é escolhido o centro geométrico do conjunto final, ou seja, é a média dos pontos de centroide ponderada pelas áreas.

- Centro dos máximos: sugere tomar como valor de saída o ponto central dos máximos. É a média ponderada, porém, são considerados somente os valores de maior pertinência.

- Média dos Máximos: o valor final é resultado do valor médio dos valores centrais ativados pelas regras.

\subsection{Modelos de regressão}

Nas atividades de avaliação imobiliária, como os imóveis são heterogêneos e há diversas características importantes a serem consideradas simultaneamente, é necessário utilizar uma técnica para realizar o ajustamento das diferenças entre os imóveis. Uma das técnicas mais utilizadas atualmente pelos avaliadores e recomendada pela NBR 14.653-2 (ABNT, 2011b) para esta função é a análise de regressão múltipla, que busca um modelo de segmento do mercado em questão, validado estatisticamente e posteriormente utilizado na projeção do valor do imóvel 
(González, 2002).

A análise de regressão é uma técnica estatística indicada para estudar o relacionamento entre as variáveis (dependentes e independentes), além de predizer o valor de uma variável específica com base em uma coleção de variáveis (Johnson e Wichern, 2002). Na avaliação imobiliária o objetivo dessa análise é obter um modelo estatístico que relaciona a variável dependente, valor do bem, com as variáveis independentes, aquelas que influenciam na formação do valor do imóvel. A Equação 1 de regressão tem como forma geral:

$$
Y=\beta_{0}+\beta_{1} X_{1}+\beta_{2} X_{2}+\ldots+\beta_{p} X_{p}+\varepsilon .
$$

Em que $\mathrm{Y}$ é a variável dependente ou a variável de interesse; $\mathrm{X}_{1}, \mathrm{X}_{2}, \ldots, \mathrm{X}_{\mathrm{p}}$ são as variáveis independentes ou explicativas; $\beta_{0}, \beta_{1}, \beta_{2}, \ldots, \beta_{\mathrm{p}}$ são os coeficientes da regressão ou regressores que representam o peso que cada variável explicativa têm na formação do valor $\mathrm{Y}$, geralmente estimados pelo Método dos Mínimos Quadrados, no qual se buscam aqueles que proporcionam a menor soma dos quadrados dos resíduos.

Segundo Johnson e Wichern (2002), quando são gerados modelos de regressão, é necessário verificar alguns pressupostos estatísticos (verificação de normalidade dos resíduos; homocedasticidade, ou seja, analisar se os resíduos possuem uma variabilidade constante e com isso um bom comportamento aleatório e não tendencioso; independência dos resíduos; ausência de pontos atípicos e ausência de multicolinearidade entre as variáveis independentes), para saber se o modelo obtido é realmente adequado para explicar a variável resposta em questão.

Gerado o modelo de regressão a partir de uma amostra, a explicação do mesmo pode ser aferida pelo seu coeficiente de determinação $\mathrm{R}^{2}$ (ABNT, 2011b). O coeficiente de determinação é dado pela razão da Soma de Quadrados da Regressão (SQR) com a Soma de Quadrados Total (SQT), como mostra a Equação 2. Como este coeficiente sempre cresce com o aumento do número de variáveis independentes e não considera o número de graus de liberdade perdidos a cada parâmetro estimado, é recomendável considerar também o coeficiente de determinação ajustado, $\boldsymbol{R}_{A j}^{2}$, Equação 3 .

$$
\begin{gathered}
R^{2}=\frac{S Q R}{S Q T}=\frac{\sum_{i=1}^{n}\left(\hat{y}_{i}-\bar{y}\right)^{2}}{\sum_{i=1}^{n}\left(y_{i}-\bar{y}\right)^{2}} \quad 0<R^{2}<1, \\
R_{A j}^{2}=1-\frac{\frac{S Q R}{(n-p)}}{\frac{S Q T}{(n-1)}} .
\end{gathered}
$$

Em que $n$ é o número de elementos da amostra, $p$ é o número de parâmetros no modelo, $\mathrm{SQR}$ é a Soma de Quadrados da Regressão e SQT é a Soma de Quadrados Total. 


\section{Determinação dos valores da avaliação imobiliária utilizando Lógica fuzzy}

Os dados utilizados neste trabalho foram disponibilizados por Dalaqua (2007) e Dalaqua, Amorim e Flores (2010) e referem-se aos valores (em reais) das transações imobiliárias de terrenos ocorridas no ano de 2006, na cidade de Álvares Machado-SP. Essas informações foram coletadas a partir de consultas às imobiliárias e entrevistas realizadas com os compradores e vendedores dos imóveis.

A metodologia não foi aplicada com base nos valores reais de compra e venda dos imóveis, mas em relação ao Valor do Metro Linear da Testada Corrigida (VMLTC), que é dado pelas Equações 4 e 5 .

$$
\begin{array}{r}
\text { VMLTC }=\frac{\text { valor_imovel }}{\text { testada_corrigida }}, \\
\text { testada_corrigida }=\sqrt{\frac{T_{\varepsilon} \times A_{\varepsilon}}{P_{p}}} .
\end{array}
$$

Em que $T_{e}$ representa o valor da testada efetiva, em metros; $A_{e}$ é a área do lote em metros quadrados e $P_{p}$ refere-se à profundidade padrão (metros) adotada pela administração municipal.

A amostra selecionada na área de interesse é composta por um total de 77 elementos, dos quais seis desses, espacialmente bem distribuídos na região, foram selecionados como pontos de verificação com o objetivo de realizar uma análise de qualidade dos resultados obtidos.

Na avaliação imobiliária, são consideradas as variáveis que caracterizam os imóveis envolvidos na amostra. Dessa forma, dos dados fornecidos por Dalaqua (2007), foram utilizadas as seguintes variáveis:

- Distância (T_DIST): representa a distância euclidiana, em metros, do terreno ao centro da cidade.

- Topografia (T_TOPO): indica o tipo de relevo do terreno (plano, aclive, declive, declive acentuado, irregular).

- Padrão construtivo (T_PADRAO): identifica a localização do imóvel quanto ao padrão construtivo da região em que está inserido (baixo, médio-baixo, médio, médio-alto, alto).

- Zona comercial (ZONA_COMER): identifica se o imóvel está localizado ou não em zona comercial.

- $\quad$ Posição (X, Y): indica a posição em coordenadas UTM (E,N) do imóvel.

A partir da amostra descrita e, de acordo com o esquema mostrado na Figura 1, a aplicação da lógica fuzzy foi feita utilizando a ferramenta Fuzzy Logical Toolbox do software Matlab 5.3 por meio das seguintes etapas:

a) Fuzzyficação das entradas, determinando o grau de pertinência de cada variável em relação a cada conjunto fuzzy, através das funções de pertinência.

b) Aplicação dos operadores fuzzy AND ou OR, por meio da determinação das regras gerais.

c) Escolha do método para a agregação de todas as saídas.

d) Escolha do método de defuzzificação para obter um valor numérico que represente a saída do 
sistema.

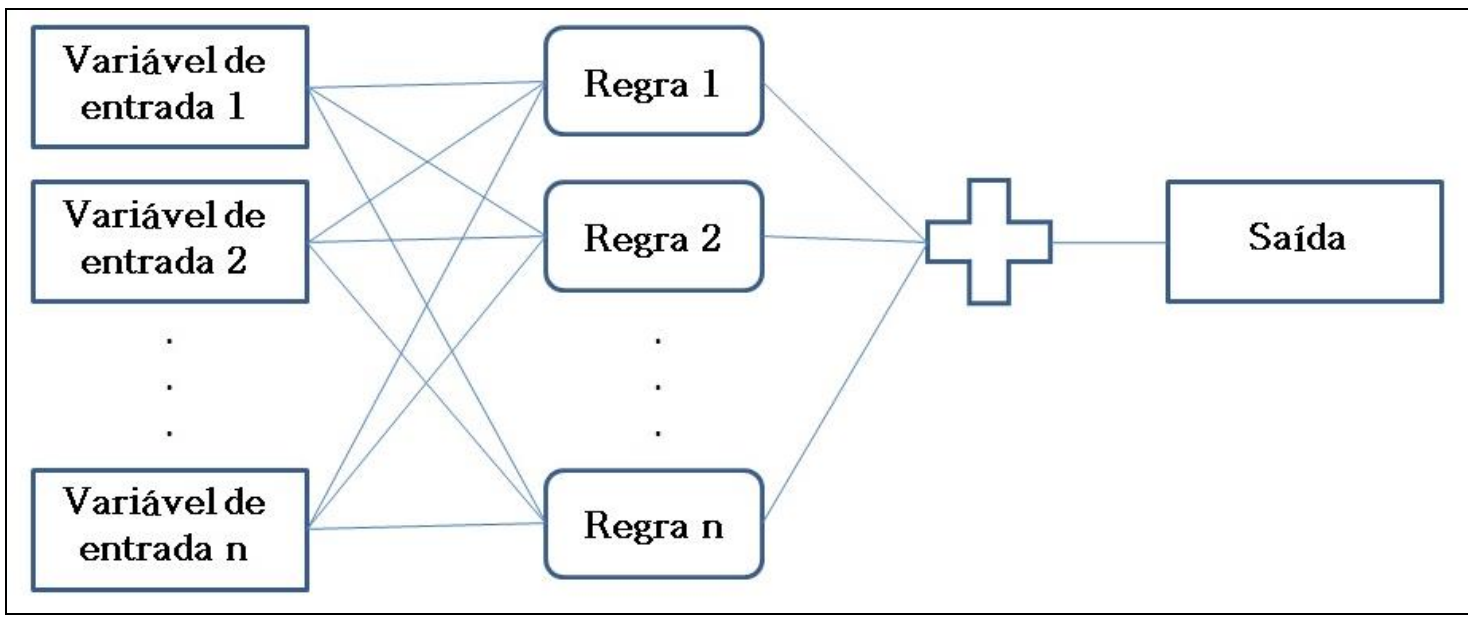

Figura 1: Esquema para a modelagem do sistema fuzzy.

Para que pudessem ser submetidos às operações de conjuntos fuzzy, os dados discretos foram transformados em variáveis linguísticas, ou seja, para cada variável foram atribuídos termos linguísticos representando os valores numéricos da mesma. A relação entre os termos e os valores foi estabelecida por um conjunto fuzzy, no qual as funções de pertinência modelaram essa relação.

Dentre as funções de pertinência disponíveis, escolheu-se a trapezoidal para a modelagem de todas as variáveis. A função trapezoidal possibilita, para um conjunto de termos e valores, estabelecer patamares constantes no centro e variações lineares nas extremidades.

Observando os intervalos definidos para a variável T_DIST na Figura 2, é possível perceber a principal característica da lógica fuzzy, o princípio da nebulosidade. Na Figura 2 (a), destacam-se as distâncias entre 400 e $500 \mathrm{~m}$, que podem ser entendidas tanto como perto, quanto como médio, com distintos graus de pertinência, e as distâncias entre 900 e $1000 \mathrm{~m}$, que podem ser entendidas tanto como médio, quanto como longe. Na Figura 2 (b), a seta indica que, para as duas funções, tem-se a mesma pertinência, 0,5. Na Figura 2 (c), nota-se que, para um mesmo valor da variável T_DIST, atribui-se a pertinência 0,75 para perto e 0,25 para médio. A Figura 2 (d) apresenta as pertinências 1 para perto e 0 para médio.

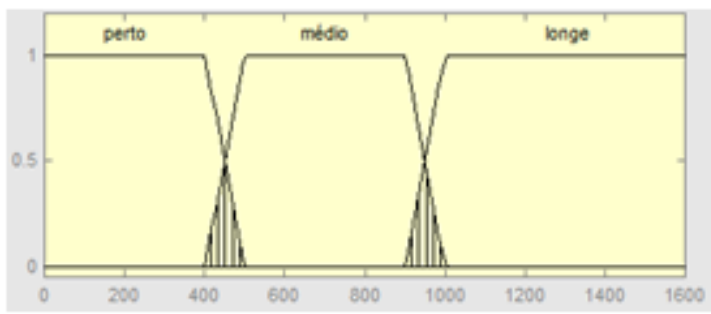

(a)

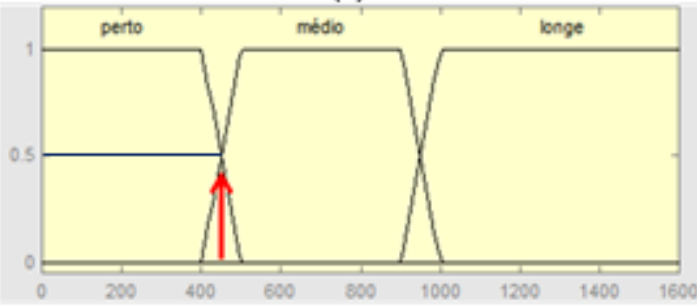

(b)

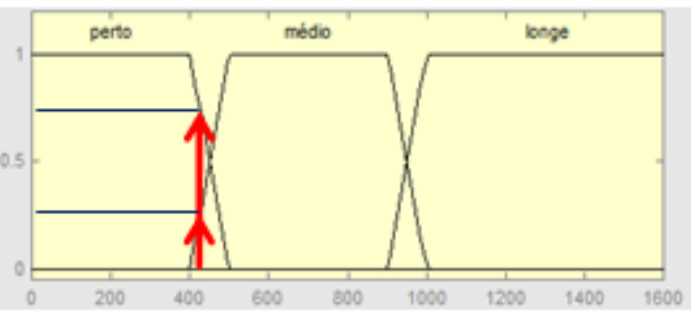

(c)

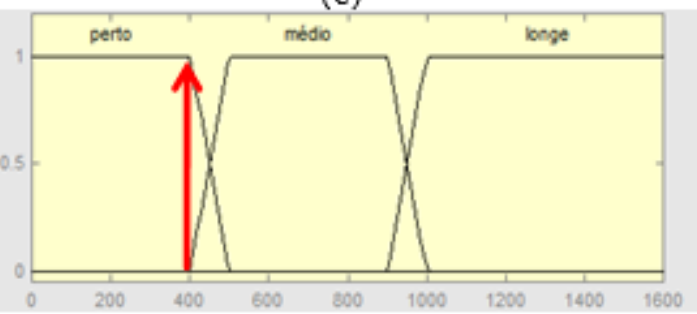

(d)

Figura 2: Princípio da nebulosidade. 
Definidas as variáveis linguísticas e as funções de pertinência para todas as variáveis, a próxima etapa consistiu na determinação das regras de inferência. $\mathrm{O}$ estabelecimento das regras foi feito com base na amostra disponível da realidade do mercado e no raciocínio lógico. Foram estabelecidas sessenta e nove regras, sendo as vinte primeiras mais simples, por exemplo:

- se (dist é perto) então (valor é alto), ou seja, se o terreno está localizado no centro da cidade ou próximo dele seu valor é considerado alto.

- se (padrão é baixo) então (valor é baixo), ou seja, se o padrão construtivo dos imóveis próximos ao terreno que está sendo avaliado for baixo o valor dele também é considerado baixo.

- se (zona_comer é sim) então (valor é alto), ou seja, se o terreno está localizado na zona comercial do município o seu valor é considerado alto.

As restantes mais complexas, como, por exemplo:

- se (dist é longe) e (padrão é baixo) e (topo é declive) e (zona é não) e (x é periferia-esquerda) e (y é periferia-superior) então (valor é baixo), ou seja, se o terreno é longe do centro da cidade, está localizado em uma região onde o padrão dos imóveis é baixo, possui uma topografia caracterizada como declive, não está localizado na zona comercial e está localizado nas periferias do município, então o valor desse terreno é baixo.

- se (dist é longe) e (padrão é médio) e (topo é aclive) e (zona é não) e (x é periferia-direita) e (y é centro), então (valor é médio-baixo), ou seja, se o terreno é longe do centro da cidade, está localizado em uma região onde o padrão dos imóveis é médio, possui relevo caracterizado como aclive, não está localizado na zona comercial, está localizado na periferia esquerda e localizado ao centro, então o valor desse terreno é médio-baixo.

- se (dist é perto) e (padrão é alto) e (topo é aclive) e (zona é sim) e (x é periferia-esquerda) e (y é centro), então (valor é alto), ou seja, se o terreno é perto do centro da cidade, está localizado em uma região onde o padrão dos imóveis é alto, possui relevo caracterizado como aclive, está localizado na zona comercial, está localizado na periferia direita e ao centro, então o valor desse terreno é alto.

Em uma representação utópica da realidade, seria necessário um modelo com 1.080 regras ( $3 \times 5 \times 4 \times 2 \times 3 \times 3)$, contudo, neste estudo foram utilizadas 69 regras para se construir o modelo fuzzy. A razão para a utilização de um número de regras reduzido está na quantidade de dados presentes na amostra utilizada, 71 pontos. Dessa forma, para validar o modelo apresentado, o conjunto de 69 regras, entre simples e complexas, se torna suficiente para cobri-los, já que há pontos que se encaixam na mesma regra e, portanto, não precisam ser repetidas.

Elaboradas as regras, definiram-se os métodos de implicação, agregação e defuzzificação, completando o sistema fuzzy e tornando-o apto para a inferência dos valores, como mostra a Figura 3. 


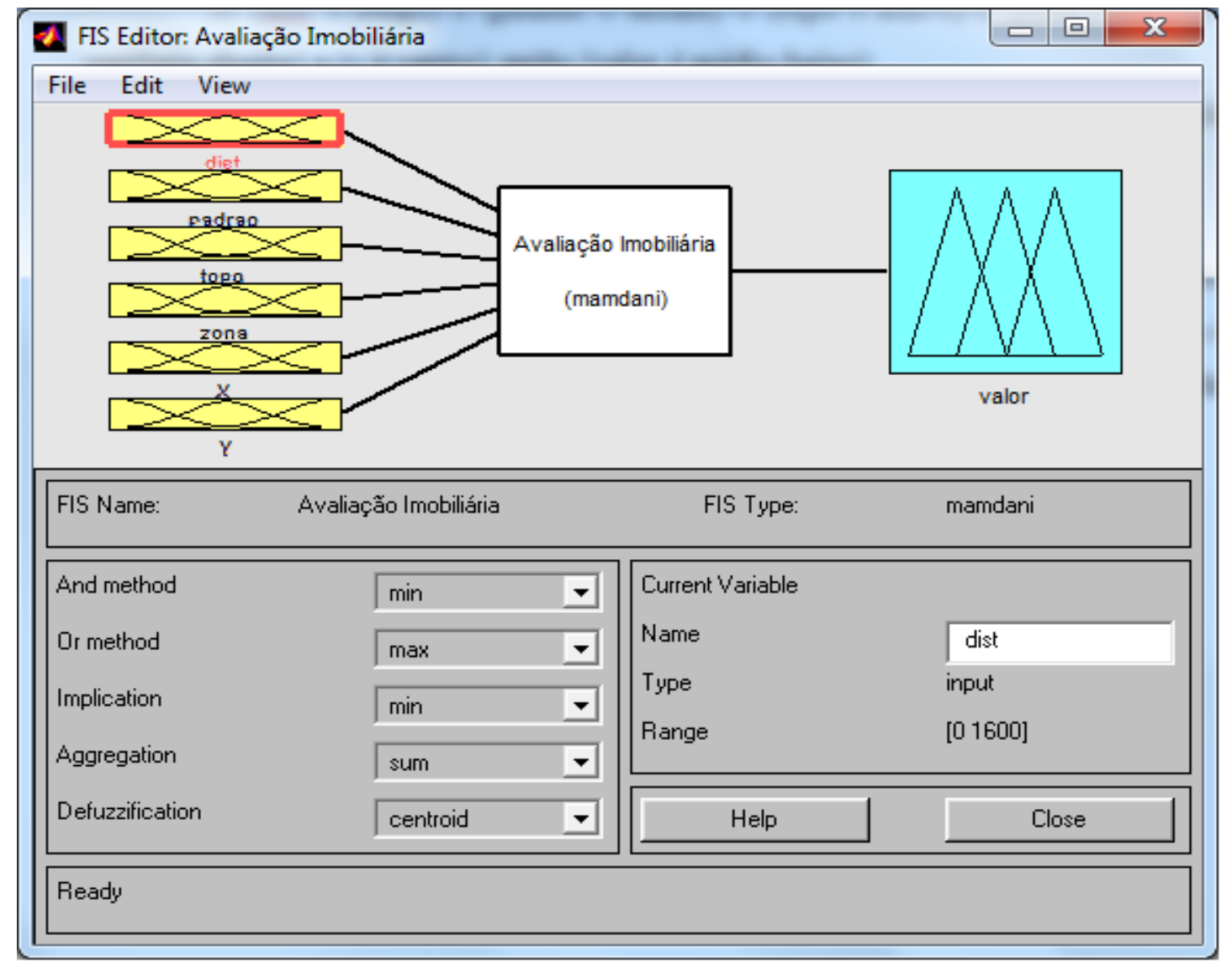

Figura 3: Métodos de implicação, agregação e defuzzificação.

Foram inferidos os valores dos 71 pontos da amostra e dos 6 pontos de verificação. A Figura 4 mostra um exemplo em que são dados os valores das variáveis de entrada e o sistema fornece a saída em valor.

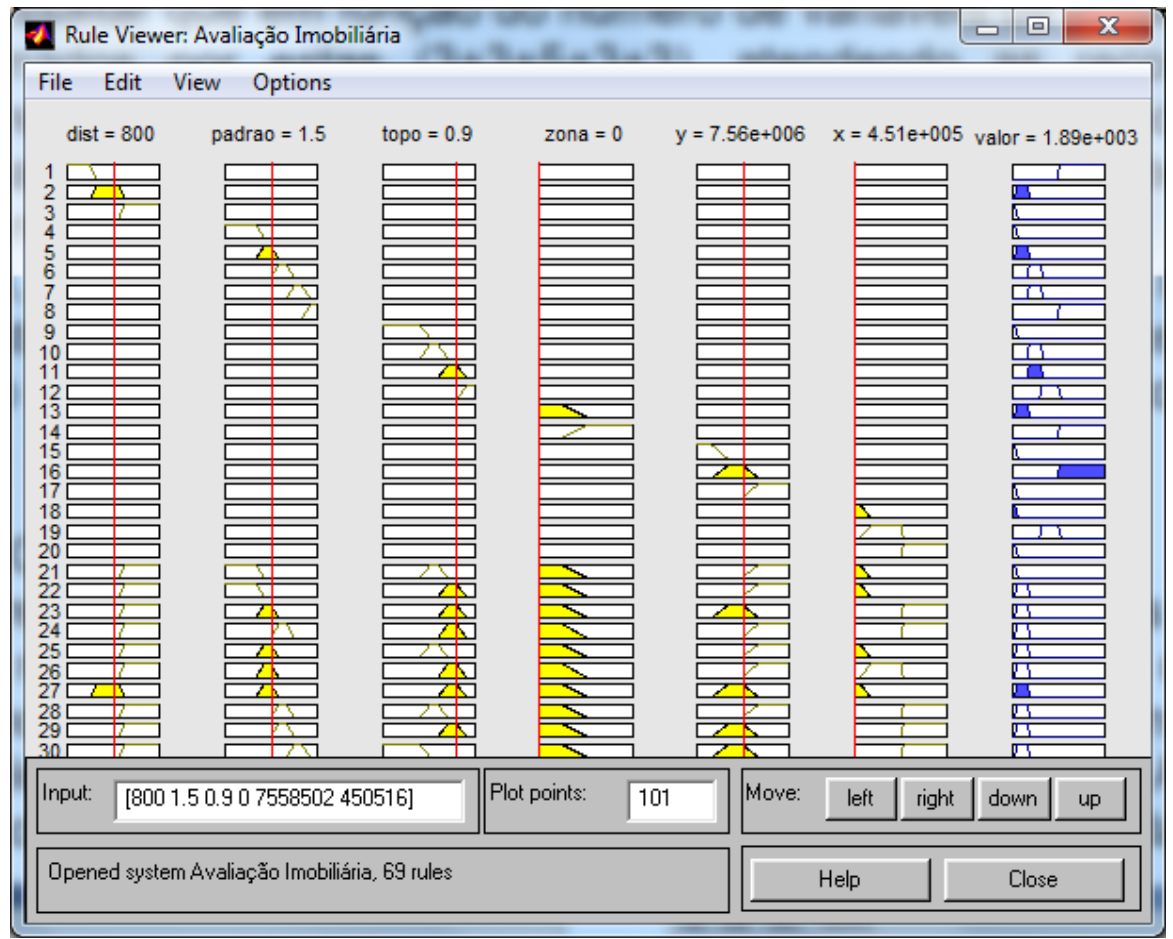

Figura 4: Exemplo de inferência de um valor. 
Para a avaliação dos resultados obtidos, foi utilizada a Raiz do Erro Médio Quadrático (REMQ), Equação 6, dos resíduos em duas situações:

- $\quad$ Situação 1: utilizando os 71 pontos da amostra;

- $\quad$ Situação 2: utilizando 6 pontos de verificação.

$$
R E M Q=\sqrt{\frac{1}{n-k-1} \sum_{i=1}^{n}\left(V_{1} M L T C \text { conhecialo }- \text { VMLTC estimadio }\right)^{2}} .
$$

Os REMQs obtidos foram R $\$ 420,55$ para a amostra de 71 pontos e $\mathrm{R} \$ 344,61$ para os 6 pontos de verificação.

Além do cálculo do REMQ, os resultados foram comparados com aqueles obtidos pelo método convencional, utilizando modelos de regressão, extraídos de Malaman et al. (2012), no qual se utilizaram modelos de regressão para o cálculo do VMLTC com o mesmo conjunto de dados. Nesse trabalho, foram estimados e comparados distintos modelos de regressão a fim de encontrar aquele que melhor representasse a variável resposta, o VLMTC.

O modelo de regressão utilizado é dado pela Equação 7, e os REMQs obtidos com este modelo foram $\mathrm{R} \$ 266,80$ para a amostra de 71 pontos e $\mathrm{R} \$ 288,29$ para os 6 pontos de verificação.

VMLTC $=846-0,00153 * \mathrm{~T} \_$DIST $+3,57 * \mathrm{~T}$ TOPO $+1,74 * \mathrm{~T}$ PADRAO + 2,89*ZONA_COMER-0,000223*X_COORD-0,000098*Y_COORD.

A Tabela 1 mostra a comparação entre os REMQs calculados com os resultados dos modelos fuzzy e com os resultados dos modelos de regressão.

Tabela 1: Comparação entre resultados obtidos com modelos fuzzy e modelos de regressão.

\begin{tabular}{c|c|c|c|c}
\hline Método & $\begin{array}{c}\text { REMQ } \\
\text { Amostra }\end{array}$ & $\begin{array}{c}\text { REMQ } \\
\text { Pontos de } \\
\text { verificação }\end{array}$ & $\begin{array}{c}\text { (REMQ Amostra } \\
\text { - REMQ Pontos } \\
\text { de verificação) }\end{array}$ & $\begin{array}{c}\text { (REMQ Amostra } \\
\text { - REMQ Pontos } \\
\text { de verificação) \% }\end{array}$ \\
\hline FUZZY & $\mathrm{R} \$ 420,55$ & $\mathrm{R} \$ 344,61$ & $-\mathrm{R} \$ 75,94$ & $22,03 \%$ \\
\hline REGRESSÃO & $\mathrm{R} \$ 266,80$ & $\mathrm{R} \$ 288,29$ & $+\mathrm{R} \$ 21,49$ & $7,50 \%$ \\
\hline
\end{tabular}

Na Tabela 1, as últimas colunas mostram a diferença entre a média da amostra e os pontos de verificação, ou seja, o quanto o modelo consegue se aproximar de valores considerados como verdadeiros. Os resultados obtidos pelo modelo de regressão, neste experimento, apresentaram resíduos menores do que os resíduos obtidos pelo modelo fuzzy. No entanto, seria precipitada uma conclusão de que o modelo de regressão utilizado seja melhor do que o modelo fuzzy, necessitando ainda de muitos experimentos e, principalmente, com uma amostra maior do que a que foi utilizada neste trabalho.

Além disso, o método proposto pode ser aperfeiçoado a partir da realização de estudos mais aprofundados quanto às funções de pertinência, regras de inferência e métodos de defuzzificação. 


\section{Análise dos resultados e discussão}

Os modelos obtidos por lógica fuzzy diferem dos modelos utilizados em avaliação imobiliária, como os modelos de regressão linear, pois não se baseiam em raciocínio indutivo e modelos matemáticos, mas sim no raciocínio dedutivo e na utilização de regras gerais para a inferência de casos particulares. Com isso, enquanto na análise de regressão são necessários dados ou informações para a geração do modelo, na lógica $f u z z y$ as regras são estabelecidas a partir de informações conhecidas, que podem ser desde os dados propriamente ditos até pré-conceitos formados com o conhecimento profissional. Além disso, os sistemas fuzzy fornecem respostas para as entradas plausíveis de serem entendidas pelas regras estabelecidas.

Ainda que os resultados não tenham sido melhores, neste caso, a utilização dos modelos fuzzy na avaliação de imóveis pode ser vantajosa e trazer melhorias para as técnicas existentes, já que não se trata de uma ciência exata e sim da estimação de valores de propriedades, na qual, na maioria das vezes, as informações são difusas e incompletas. Por exemplo, um terreno pode estar inserido tanto na faixa de padrão alto quanto na faixa de padrão médio, com diferentes graus de pertinência. Dessa forma, não é necessário definir, com certeza, onde aquele terreno está inserido.

\section{Conclusões}

Após a comparação entre os resultados obtidos, a partir da Regressão Linear e da Lógica Fuzzy, notou-se que não houve grande melhora com a aplicação da lógica fuzzy. No entanto, este método pode ser útil para lidar com variáveis que contenham ambiguidades, informações incompletas, incertezas nas definições de seus valores e ainda permitir as representações de estratégias das partes. O método proposto para avaliação imobiliária, por meio de modelos fuzzy, pode contribuir com as técnicas atuais, principalmente pela simplicidade e clareza com que os dados são processados e os resultados apresentados. Ressalva-se que, para aplicação desse método, deve ser utilizada uma amostra maior do que a utilizada neste trabalho, fato que poderá melhorar significativamente os resultados.

O método proposto não se enquadra nos métodos estabelecidos pelas NBR 13.653-2 (ABNT, 2011b), mas é uma nova proposta que pode trazer contribuições e melhorias a essa atividade. Os sistemas baseados em lógica fuzzy tentam simular o pensamento humano e são bastante aplicados e recomendados para situações duvidosas e imprecisas como as variáveis estudadas na avaliação imobiliária, especialmente as variáveis qualitativas que se apresentam bastante subjetivas.

Para trabalhos futuros, recomenda-se a utilização de mais regras de inferência e mais variáveis, um estudo mais aprofundado sobre as funções de pertinência para a problemática em questão e o melhor método de defuzzificação. Outra possibilidade é a utilização de diferentes softwares, já que só foi testado o Matlab, desconhecendo as funcionalidades de outros, por exemplo, o FuzzyTech. Além disso, podem ser utilizados métodos não supervisionados (guiados pelos dados), tais como Redes Neurais.

Outro fator a ser explorado é a utilização de lógica fuzzy para a elaboração da PVG, utilizando Sistemas de Informação Geográfica, de forma que os dados fiquem armazenados em um banco de dados possibilitando melhor aproveitamento dos mesmos para atualizações futuras. 


\section{AGRADECIMENTOS}

Os autores agradecem à Coordenação de Aperfeiçoamento de Pessoal de Nível Superior (CAPES) pela bolsa de mestrado concedida durante o desenvolvimento deste trabalho.

\section{REFERÊNCIAS BIBLIOGRÁFICAS}

ABNT (Associação Brasileira de Normas Técnicas). NBR 14.653-1: Avaliação de bens - Parte 1: Procedimentos Gerais. Rio de Janeiro: ABNT, 2011a.

ABNT (Associação Brasileira de Normas Técnicas). NBR 14.653-2: Avaliação de bens - Parte 2: imóveis urbanos. Rio de Janeiro: ABNT, 2011b.

Averbeck, Carlos E. Os sistemas de cadastro e planta de valores no município: prejuízos da desatualização. Dissertação (Mestrado em Engenharia Civil) - Curso de Pós-Graduação em Engenharia Civil, Universidade Federal de Santa Catarina - UFSC, Florianópolis, SC. 2003. 200p.

Azevedo, Fernando M.; Brasil, L. M.; Oliveira, R. C. L. Redes Neurais - Com aplicações em Controle e sem Sistemas Especialistas. Bookstore Livraria Ltda. Florianópolis - SC. 2000. 190p.

Barros, Laécio C.; Bassanezi, R. C. Tópicos de lógica Fuzzy e biomatemética. Campinas: Unicamp, 2006.

Benini, Luiz C.; Meneguette Junior, M. Uma abordagem para modelagem de dados com o uso de sistemas neuro-Fuzzy: aplicações geoespaciais. In: Barbelos, C. A. Z. ; Andrade, E. X. L. ; Boaventura, M. (Ed.). XXXII CNMAC. São Carlos: SBMAC, 2009, (Notas em Matemática Aplicada, v. 43).

Brasil. Lei n ${ }^{\circ}$ 10.257, de 10 de julho de 2001. Regulamenta os artigos. 182 e 183 da Constituição Federal e estabelece diretrizes gerais da política urbana e dá outras providências. Brasília. 2001.

Brasil. Lei complementar $n^{\circ} 101$, de 4 de maio de 2000.Estabelece normas de finanças públicas voltadas para a responsabilidade na gestão fiscal e dá outras providências. Brasília, DF, 04 mai. 2000.

Cay, Tayfun ; Iscan, F. Fuzzy expert system for land reallocation in land consolidation. Expert Systems with Applications, Turkey, v. 38, n. 9, p. 1105-11071, 2011.

Dalaqua, Roberto R. Aplicação de métodos combinados de avaliação imobiliária na elaboração da planta de valores genéricos. Dissertação (Mestrado em Ciências Cartográficas) - Curso de Pós-Graduação em Ciências Cartográficas, Faculdade de Ciências e Tecnologia da Universidade Estadual Paulista - UNESP, Presidente Prudente, SP. 2007, 128p.

Dalaqua, Roberto R.; Amorim, A.; Flores, E. F. Utilização de métodos combinados de avaliação imobiliária para a elaboração da Planta de Valores Genéricos. Boletim de Ciências Geodésicas, v.16, p.232-253, 2010.

González, Marco A. S. Aplicação de Técnicas de Descobrimento de Conhecimento em Bases de Dados e de Inteligência Artificial em Avaliação de Imóveis. Tese (Doutorado em Engenharia Civil) - Programa de Pós-Graduação em Engenharia Civil, UFRGS, Porto Alegre - RS. 2002. 
Johnson, Richard A.; Wichern, D. W. Applied Multivariate Statistical Analysis. New Jersey: Prentice-Hall, 2002. $5^{\text {a }}$ Ed.

Malaman, Carolina S. ; Marques, A. P. S. ; Tachibana, V. M. ; Amorim, A. Elaboração de planta de valores genéricos a partir de distintos modelos de regressão linear múltipla. In: IV Simpósio Brasileiro de Ciências Geodésicas e Tecnologias da Geoinformação. Anais. Recife. 2012.

Mello, Renato; Santello, R. O uso de Lógica Fuzzy para Avaliação de imóveis e exemplos de aplicação. Revista Produção Online, Florianópolis, SC, v.12, n.4, p.904-927, 2012.

Moraes, Odair B. Método de análise de dados para avaliação de áreas urbanas recuperadas: Uma abordagem utilizando a lógica Fuzzy. (Tese de Doutorado). Escola Politécnica da USP, São Paulo. 2008 .

Murgel Filho, Wolmar. Sistema Nebuloso de apoio à produção de planta de valores. Dissertação (Mestrado em Engenharia de Computação - Geomática) - Curso de Pós-Graduação em Engenharia de Computação, Universidade do Estado do Rio de Janeiro - UERJ, Rio de Janeiro, RJ. 2005, 132p.

Ortega, Neli R. S. Aplicação da Teoria de Conjuntos Fuzzy a Problemas da Biomedicina. São Paulo. Tese (Doutorado em Física). Instituto de Física da Universidade de São Paulo. 2001. 152 p.

Runkler, Thomas A. Selection of appropriate defuzzification methods using application specific properties. In: IEEE Transactions on Fuzzy Systems, 1997.

Recebido em 18 de novembro de 2015.

Aceito em 11 de agosto de 2016. 